\title{
Homogenization Fundamentals
}

\author{
Poonam Dhankhar \\ M.Tech (Food tech), G.J.U.S \& T, Hissar
}

\begin{abstract}
Homogenization is a process of achieving homogeneity through out a product by particle size modification. it is characterized into three major categories:-ultrasonic pressure homogenization, pressure homogenization mechanical homogenizer. Application of homogenization are diverse in food industry, chemical industry, pharmaceutical and chemical industry. Most important use of homogenization process in food industry is in milk homogenization. Selection criteria of homogenizers depends on particular application.
\end{abstract}

\section{INTRODUCTION}

Homogeneity and heterogeneity are concepts relating to the uniformity or lack thereof in a substance. A material that is homogeneous is uniform in composition or character; one that is heterogeneous lacks uniformity in one of these qualities. The concepts are applicable to combinations at every level of complexity, from atoms to populations of animals or people, to galaxies. Hence, a substance may be homogeneous on a larger scale, compared to being heterogeneous on a smaller scale within the same substance ${ }^{(1)}$

]"In 1899 Auguste Gaulin obtained a patent on his homogenizer. The patent consisted of a 3 piston pump in which product was forced through one or more hair like tubes under pressure.

At the World's Fair in Paris in 1900, Auguste Gaulin exhibited his invention... a process for "treating" milk. According to the literature and publications of that time, the word "'homogenized" was first used to describe milk treated by the Gaulin machine. Therefore, the terms "homogenization" and "homogenizer" historically relate to the process and equipment developed by Gaulin. The homogenizer basically consists of a positivedisplacement pump to which is attached a homogenizing valve assembly. The pump forces fluids through the homogenizing valve under pressure. The term "homogenization" refers to the process or action that occurs within the homogenizing valve assembly. ${ }^{(4)}$

\section{THE FIELD OF HOMOGENIZING[ ${ }^{8][9]}$}

The field of homogenizing encompasses a very broad area. The word homogenize means "to make or render homogeneous" while homogeneous means "having the same composition, structure, or character throughout". Homogenizing is what is called an umbrella word - a word which covers a very large area. When someone says that they are homogenizing, they may mean that they are actually doing one or more of the following, blending, mixing, disrupting, emulsifying, dispersing, stirring etc. ${ }^{\left({ }^{()(9)}\right.}$ The current processes or methods of homogenizing can be broken down into three (3) major categories,

- ultrasonic,

- pressure, and

- Mechanical.

\section{1) ULTRASONIC HOMOGENIZING:-}

One widely used method to disrupt cells is ultrasonic disruption. These devices work by generating intense sonic pressure waves in a liquid media. The pressure waves cause streaming in the liquid and, under the right conditions, rapid formation of micro-bubbles which grow and coalesce until they reach their resonant size, vibrate violently, and eventually collapse. This phenomenon is called cavitation. The implosion of the vapor phase bubbles generates a shock wave with sufficient energy to break covalent bonds. Shear from the imploding cavitation bubbles as well as from eddying induced by the vibrating sonic transducer disrupt cells. There are several external variables which must be optimized to achieve efficient cell disruption. These variables are as follows:

- Tip amplitude and intensity

- Temperature

- Cell concentration

- Pressure

- Vessel capacity and shape

Modem ultrasonic processors use piezoelectric generators made of lead zirconate titanate crystals. The vibrations are transmitted down a titanium metal horn or probe tuned to make the processor unit resonate at 15$25 \mathrm{kHz}$. The rated power of ultrasonic processors vary from 10 to 375 Watts. low power output does not 
necessarily mean that the cell disintegrator is less powerful because lower power transducers are generally matched to probes having smaller tips. It is the power density at the tip that counts. Higher output power is required to maintain the desired amplitude and intensity under conditions of increased load such as high viscosity or pressure. The larger the horn, the more power is required to drive it and the larger the volume of sample that can he processed. On the other hand, larger ultrasonic disintegrators generate considerable heat during operation and will necessitate aggressive external cooling of the sample. Typical maximum tip amplitudes are 30-250 urn and resultant output intensities are in the range of 200-2000 W/square $\mathrm{cm}$.

The temperature of the sample suspension should be as low as possible. In addition to addressing the usual concerns about temperature liability of proteins, low media temperatures promote high-intensity shock front propagation. So ideally, the temperature of the ultrasonicated fluid should be kept just above its freezing point. The ultrasonic disintegrator generates considerable heat during processing and this complicates matters. Disruption can also be enhanced by increased hydrostatic pressure (typically 15-60 psi) and increased viscosity, providing the ultrasonic processor has sufficient power to overcome the increased load demand and the associated sample heating problems can be solved. For microorganisms the addition of glass beads in the 0.05 to $0.5 \mathrm{~mm}$ size range enhances cell disruption by focusing energy released by the bubble implosions and by physical crushing. Beads are almost essential for disruption of spores and yeast. A good ratio is one volume of beads to two volumes of liquid. Tough tissues such as skin and muscle should be macerated first in a blender or the like and confined to a small vessel during ultrasonic treatment. The tip should not be placed so shallow in the vessel as to allow foaming. Antifoaming agents or other materials which lower surface tension should be avoided. Finally, one must keep in mind that free radicals are formed in ultrasonic processes and that they are capable of reading with biological material such as proteins, polysaccharides, or nucleic acids. Damage by oxidative free radicals can be minimized by including scavengers like cysteine, dithiothreitol, or other SH compounds in the media or by saturating the sample with a protective atmosphere of helium or hydrogen gas.

For practical reasons, the tip diameter of ultrasonic horns cannot exceed about 3 inches. This sets a limit on the scale-up of these devices. While standard sized ultrasonic disrupters have been adapted to continuous operation by placing the probe tip in a chamber through which a stream of cells flow, cooling and free radical release present problems.

\section{PRESSURE HOMOGENIZING}

High-pressure homogenizers have been used to disrupt microbial cells for many years. With the exception of highly filamentous microorganisms, the method has been found to be generally suitable for a variety of bacteria, yeast and mycelia.

This type of homogenizer works by forcing cell suspensions through a very narrow channel or orifice under pressure. Subsequently, and depending on the type of high-pressure homogenizer, they may or may not impinge at high velocity on a hard-impact ring or against another high-velocity stream of cells coming from the opposite direction. Machines which include the impingement design are more effective than those which do not. Disruption of the cell wall occurs by a combination of the large pressure drop, highly focused turbulent eddies, and strong shearing forces. The rate of cell disruption is proportional to approximately the third power of the turbulent velocity of the product flowing through the homogenizer channel, which in turn is directly proportional to the applied pressure. Thus, the higher the pressure, the higher the efficiency of disruption per pass through the machine. The operating parameters which effect the efficiency of high-pressure homogenizers are as follows:

- Pressure

- Temperature

- Number of passes

- Valve and impingement design

- Flow rate

High-pressure homogenizers have long been the best available means to mechanically disrupt nonfilamentous microorganisms on a large scale. Animal tissue also can be processed but the tissue must be pretreated with a blade blender, rotor-stator homogenizer, or paddle blender. The supremacy of high-pressure homogenizers for disruption of microorganisms is now being challenged by bead mill homogenizers. Still, in terms of throughput, the largest industrial models of high-pressure homogenizers outperform bead mills. The maximum volume of microbial suspension per hour that can be treated by the larger commercial machines is 4,500 liters for high-pressure homogenizers versus about 1,200 liters for bead mills. Even larger capacity highpressure homogenizers are available but their efficiency in disrupting microbial cells has not been documented. This throughput advantage is diminished somewhat by the fact that most high-pressure homogenizers require several passes of the cell suspension to achieve high levels of cell disruption whereas bead mills frequently need only one. 
A familiar commercial high-pressure homogenizer for the laboratory is the French press which uses a motor-driven piston inside a steel cylinder to develop pressures up to 40,000 psi. Pressurized sample suspensions up to $35 \mathrm{~m} 1$ are bled through a needle valve at a rate of about $1 \mathrm{ml} / \mathrm{min}$. Because the process generates heat, the sample, piston and cylinder are usually pre-cooled. Typical pressures used to disrupt yeast are 8,000 to 10,000 psi and several passes through the press may be required for high efficiency of disruption. Generally, the higher the pressure, the fewer the passes. Pressure cells rated at 20,000 psi maximum come in capacities of 3.7 and $35 \mathrm{~m} 1$ and there is also a $35 \mathrm{~m} 1$ capacity cell rated at 40,000 psi.

Most high-pressure homogenizers used for homogenization were adapted from commercial equipment designed to produce emulsions and homogenates in the food and pharmaceutical industries. They combine high pressure with an impingement valve. Those with a maximum pressure rating of 10,000 psi rupture about $40 \%$ of the cells on a single pass, $60 \%$ on the second pass, and $85 \%$ after four passes. Capacities of continuous homogenizers vary from 55 to 4,500 liters/hr at $10-17 \% \mathrm{w} / \mathrm{v}$ cell concentrations. With the larger capacity machines several passes are needed to achieve high yields of disruption. Considerable heat can be generated during operation of these homogenizers and therefore a heat exchanger attached to the outlet port is essential.

\section{MECHANICAL HOMOGENIZERS}

Mechanical homogenizers can be broken down into two (2) separate categories,

a) rotor-stator homogenizers and

b) blade type homogenizers.

\section{a) ROTOR-STATOR HOMOGENIZERS}

Rotor-stator homogenizers (also called colloid mills or Willems homogenizers) generally outperform cutting blade-type blenders and are well suited for plant and animal tissue. Combined with glass beads, the rotor-stator homogenizer has been successfully used to disrupt microorganisms. However, the homogenized sample is contaminated with minute glass and stainless steel particles and the abrasive wear to the rotor-stator homogenizer is unacceptably high. Cell disruption with the rotor-stator homogenizer involves hydraulic and mechanical shear as well as cavitation. Some people in the homogenizing field also claim that there is to a lesser extent high-energy sonic and ultrasonic pressure gradients involved.

While some do not believe in the theory that high-energy sonic and ultrasonic pressure gradients are involved with mechanical homogenizers. The only thing that ultrasonic and mechanical (rotor-stator) homogenizing have in common is that both methods generate and use to some degree cavitation. Cavitation is defined as the formation and collapse of low-pressure vapor cavities in a flowing liquid. Cavitation is generated as you move a solid object through a liquid at a high rate of speed. In ultrasonics the object being moved is the probe which is being vibrated at a very high rate of speed generating cavitation. In mechanical homogenizing (rotor-stator) the blade (rotor) is being moved through the liquid at a high rate of speed generating cavitation.

The rotor-stator generator type homogenizer was first developed to make dispersions and emulsions, and most biological tissues are quickly and thoroughly homogenized with this apparatus. Appropriately sized cellular material is drawn up into the apparatus by a rapidly rotating rotor (blade) positioned within a static head or tube (stator) containing slots or holes. There the material is centrifugally thrown outward in a pump like fashion to exit through the slots or holes. Because the rotor (blade) turns at a very high rpm, the tissue is rapidly reduced in size by a combination of extreme turbulence, cavitation and scissor like mechanical shearing occurring within the narrow gap between the rotor and the stator. Since most rotor-stator homogenizers have an open configuration, the product is repeatedly recirculated. The process is fast and depending on the toughness of the tissue sample, desired results will usually be obtained in 15-120 seconds. For the recovery of intracellular organelles or receptor site complexes, shorter times are used and the rotor speed is reduced. The variables to be optimized for maximum efficiency are as follows:

- Design and size of rotor-stator (generator)

- Rotor tip speed

- Initial size of sample

- Viscosity of medium

- Time of processing or flow rate

- Volume of medium and concentration of sample

- Shape of vessel and positioning of rotor-stator

The size of the rotor-stator probe (also called generator) can vary from the diameter of a pencil for $0.01-10 \mathrm{mI}$ sample volumes to much larger units having batch capacities up to 19,000 liters or, for on-line units, capabilities of 68,000 liters/hr. Rotor $\mathrm{s}^{\mathrm{p}}$ eeds vary from $3,000 \mathrm{rpm}$ for large units to $8,000-60,000 \mathrm{rpm}$ for the smaller units. In principle, the rotor speed of the homogenizer should be doubled for each halving of the rotor diameter. It is not the rpm's of the motor but the tip velocity of the rotor that is the important operating 
parameter. Other factors such as rotor-stator design, which there are many, materials used in construction, and ease of leaning are also important factors to consider in selecting a rotor-stator homogenizer.

Laboratory size rotor-stator homogenizers process liquid samples in the $0.01 \mathrm{ml}$ to 20 liter range. The capacity of the rotor-stator should be matched to the viscosity and volume of the medium and with the type and amount of plant and animal tissue to be processed. The speed and efficiency of homogenization is greatly degraded by using too small a homogenizer, and the volume range over which a given homogenizer rotor-stator size will function efficiently is only about 10 fold. Also, most of the laboratory-sized homogenizers function properly only with liquid samples in the low to medium viscosity range $(<10,000 \mathrm{cps})$. This must be balanced against the practical observation that concentratedsamples, by colliding more frequently, are broken up more rapidly. Higher viscosity samples can be processed but require specially shaped homogenization vessels or unique rotor-stator configurations. The size of the sample prior to processing with the homogenizer must be small enough to be drawn inside the stator. Therefore, samples often must be pre-chopped, cut or fragmented.

Foaming and aerosols can be a problem with rotor-stator homogenizers. Keeping the tip of the homogenizer well submerged within the media and the use of properly sized vessels helps with the first problem. Squareshaped or fluted vessels give better results than round vessels and it is also beneficial to hold the immersed tip off center. Aerosols can be minimized by using covered vessels.

There are no aerosols with in-line homogenizers. Even though a number of the laboratory rotor-stator homogenizers use sealed motors, none of them are truly explosion-proof. Due caution should be followed when using flammable organic solvents by conducting the homogenization in a well ventilated hood. On the positive side, rotor-stator homogenizers generate minimal heat during operation and this can be easily dissipated by cooling the homogenization vessel in ice water during processing.

The larger rotor-stator homogenizers are either scaled up versions of the laboratory models or in-line homogenizers. The latter contain teeth on the edge of a horizontally oriented, multi-bladed, high-speed impeller aligned in close tolerance to matching teeth in a static liner.

\section{b) BLADE TYPE HOMOGENIZERS:-}

Although less efficient than rotor-stator homogenizers, blade homogenizers (also called blenders) have been used for many years to produce fine brie and extracts from plant and animal tissue. The cutting blades on this class of homogenizer are either bottom or top driven and rotate at speeds of 6,000 to 50,000 rpm. Blenders are not suitable for disruption of microorganisms unless glass beads or other abrasives are added to the media and then one encounters the same problems as were mentioned above for rotor-stator homogenizers. Many plant tissue homogenizers undergo enzymatic browning which is a biochemical oxidation process which can complicate subsequent separation procedures. Enzymatic browning is minimized by carrying out the extraction in the absence of oxygen or in the presence of thiol compounds such as mercaptoethanol. Sometimes addition of polyethylene imine, metal chelators, or detergents such as Triton X-100 or Tween-80 also helps. Blade homogenizers are available for a range of liquid sample sizes from $0.01 \mathrm{ml}$ to multi gallons. Some of the higher $\mathrm{rpm}$ homogenizers can reduce tissue samples to a consistent particulate size with distributions as small as 4um as determined by flow cytometric analysis

\section{6) Applications of homogenization ${ }^{[3][5][6]}$}

\section{1) Food \& Dairy Applications of Homogenization}

Homogenization is the process of reducing the particle size of fluid products such as milk, fruit juice and sauces, under conditions of extreme pressure, sheer, turbulence, acceleration and impact, to make them more stable and have a better texture. The effect is achieved by forcing the product through a special homogenizing valve at a very high pressure. Particles enter the homogenizer with sizes ranging typically from $0.2-20$ microns. Large particles are dispersed to produce a product with particles ranging typically from 0.4 to 1 micron depending on the application

\section{2) Cosmetic Homogenizer Applications}

High-pressure homogenization is essential to the quality and stabilization of beauty creams, lotions, nail varnishes, shampoos, toothpastes, and emulsions containing different oils since it is a very effective way to create the exact required product texture (via particle size reduction) while at the same time creates very stable product compared to the traditional devices such as agitators, stirrers, rotor-stator devices or colloid mills. This is achieved by reducing the particle size and uniformity under conditions of extreme pressure, shear and stress. The result is a homogeneous effective product with superior stability and shelf life. It is well known fact that one of the limiting factors in the performance of some cosmetic product, for instance beauty creams, is its ability to be absorbed into the skin. Drastic particle size reduction enables the skin to absorb the active ingredients in these creams as the surface area increases after the homogenization (vs volume ratio). High-pressure 
homogenization (also known as micronization) also ensures the most efficient use of the active agents, thus reducing product costs.

\section{3) Biotechnology Homogenizer Applications}

High-pressure homogenization is widely used for cell rupture (cell rupturing, cell disruption) applications in order to extract intra-cell substances without using solvents or other chemicals for the cell wall breakage. The main purpose is to release intercellular substances such as proteins, enzymes, and vitamins that are found in high concentrations in various organisms such as yeasts, algae and bacteria (and other biological products). High-pressure homogenizers are very effective and efficient for cell rupture operations and can often maximize the yield from valuable source material while keeping product quality at a very high level..

\section{4) Pharmaceutical Homogenizer Applications}

Homogenization (known in the pharmaceutical industry as micronization) is the process of reducing the particle sizes of pharmaceutical products, under very high pressures, sheer, turbulence, acceleration and impact, to make them more stable and clinically effective. The bioavailability of the product increases and the tolerance of some otherwise borderline drugs can improve.

The emulsion, suspension or solution is pumped into the high-pressure homogenizer after which it is forced through a special homogenization valve at extremely high pressures (up to 1,500 bar / 21,750 PSI). The particles enter the homogenizer with $500 \mu \mathrm{m}$ maximum sizes, after the treatment the large particles are dispersed and reduced, the particle size typically range from 0.4 to 1 micron depending on the specific application.

High-pressure homogenization in the pharmaceutical industry has proven its ability to make more stable product, with better active ingredient dispersion, than has been achieved with conventional stirrers, rotor-stator devices or colloid mills. It achieves this by reducing the particle size and uniformity under conditions of extreme pressure.

\section{5) Homogenization in Chemical Processing}

High-Pressure Pumping and Homogenization in chemical processing receives much less publicity than its use in the food and dairy industry, but applications are many and varied. The homogenization stage is ideal to create stable emulsions, dispersions and mixes. The subdivision of particles and droplets increases their available surface area, which can improve chemical reaction processes by reducing reaction time and temperatures, and minimize or even eliminate the need for catalysts. The application of high pressure often enhances extraction processes, and the color intensity in many pigments and paints. Homogenization is used in the petrochemical industry for optimum additive blending and viscosity control.

Milk homogenization:-[2][4][7][10]

In food industry most prominent use of homogenizers is for milk homogenization.

\section{1) Objectives:-}

a) Counteracting segregation, for the most part creaming.-to achieve this, the size of the fat globules should be greatly reduced. A cream layer or sediment in the product may be a nuisance for the user, especially if the package is nontransparent.

b) improving stability towards partial coalescence.-the increased stability of homogenized fat globule is caused by the reduced diameter and by the acquired surface layer of the fat globules. Moreover, partial coalescence especially occurs in a cream layer and such a layer forms much more slowly in homogenized products. All in all, prevention of partial coalescence usually is the most important purpose of homogenization.

\section{2) homogenization effects:-}

Milk is an oil-in-water emulsion, with the fat globules dispersed in a continuous skim milk phase. If raw milk were left to stand, however, the fat would rise and form a cream layer. Homogenization is a mechanical treatment of the fat globules in milk brought about by passing milk under high pressure through a tiny orifice, which results in a decrease in the average diameter and an increase in number and surface area, of the fat globules. The net result, from a practical view, is a much reduced tendency for creaming of fat globules. Three factors contribute to this enhanced stability of homogenized milk: a decrease in the mean diameter of the fat globules (a factor in Stokes Law), a decrease in the size distribution of the fat globules (causing the speed of rise to be similar for the majority of globules such that they don't tend to cluster during creaming), and an increase in density of the globules (bringing them closer to the continuous phase) owing to the adsorption of a protein membrane. In addition, heat pasteurization breaks down the cryo-globulin complex, which tends to cluster fat globules causing them to rise. 
7.3) advantages and disadvantages:-

\begin{tabular}{|c|c|}
\hline advantages & Disadvantages \\
\hline $\begin{array}{l}\text { - Increase of the total surface of the fat } \\
\text { globule, which prevents or delays creaming of } \\
\text { the fat and increases light reflection. }\end{array}$ & $\begin{array}{l}\text { - Increased area for attack of microbial lipase, resulting in } \\
\text { lipolytic changes }\end{array}$ \\
\hline - Enhancement of taste and texture & $\begin{array}{l}\text { - Increased sensitivity to light influences, leading to taste } \\
\text { defects such as rancid, soupy or oxidized }\end{array}$ \\
\hline - Increase in digestibility, & - Increased area for microbial contamination. \\
\hline - The colour becomes whiter. & $\begin{array}{l}\text { - Reduced thermal stability of the protein (homogenization } \\
\text { must be done after the UHT treatment for UHT milk) }\end{array}$ \\
\hline - The tendency to foam increases somewhat. & $\bullet$ \\
\hline - A smoother texture appears. & $\bullet$ \\
\hline
\end{tabular}

\section{4) Homogenization Mechanism}

The theories of homogenization that have been presented over the years have attempted to relate this high-energy transition in the valve to the results observed in the product. In the past, it was difficult to prove or disprove many of these theories, but it appears that turbulence is the primary mechanism.

First, something should be said about shear in the valve, because many people assume that shear is the main mechanism of homogenization. When using the term "shear", one is describing the elongation and subsequent breakup of a dispersed phase droplet because of different fluid velocities surrounding the droplet. The classical theories of shear effects, as related to the flow profile in the homogenizing valve, are difficult to justify. Two arguments against shear are (1) the viscosity of many fats and oils is greater than the maximum value allowed for shearing of the droplets, and (2) the flow profile in the valve is such that the bulk of the liquid does not experience large velocity gradients due to the boundary layer effects.

In the cavitation theory, the liquid encounters intense cavitation because of the large pressure drop through the valve. When the pressure drop is large enough, the vapor pressure of the liquid exceeds the ambient pressure causing formation of vapor bubbles (cavities in the liquid). When the cavitation bubbles implode (collapse of the cavities), shock waves are generated in the liquid. These shock waves break apart the dispersed droplets.

The second homogenization theory, relating to turbulence, suggests that the energy dissipating in the liquid generates intense turbulent eddies.These eddies would be of the same size as the average droplet diameter. The intense energy of the turbulence and localized pressure differences would tear apart the droplets, reducing their average size. Flow visualization experiments by this author using a modeled quartz homogenizing valve revealed that, for emulsions, droplet size reduction is produced by turbulent flow. Backpressure or secondstage pressure suppresses cavitation but maintains turbulent flow. Droplets passing through the turbulent jet at the discharge from the gap are immediately disrupted by intense turbulent flow.

\section{Single stage homogenization and two stage homogenization:-}

\begin{tabular}{|c|c|}
\hline Single stage homoogenization & \\
\hline $\begin{array}{ll}- & \text { PRESSURE USED- 2500psi } \\
- & \text { MEAN SIZE- } 0.5 \mu \mathrm{m} \\
- & \text { RANGE- } 0.2-2 \mu \mathrm{m} \\
- & \text { Clustering takes place }\end{array}$ & $\begin{array}{l}\text { - } \\
\text { In } 1^{\text {st }} \text { Ptage- } 2000 \mathrm{psi} \\
\text { In } 2^{\text {nd }} \text { stage- } 500 \mathrm{psi} \\
-\quad \quad \text { No clustering takes place }\end{array}$ \\
\hline
\end{tabular}



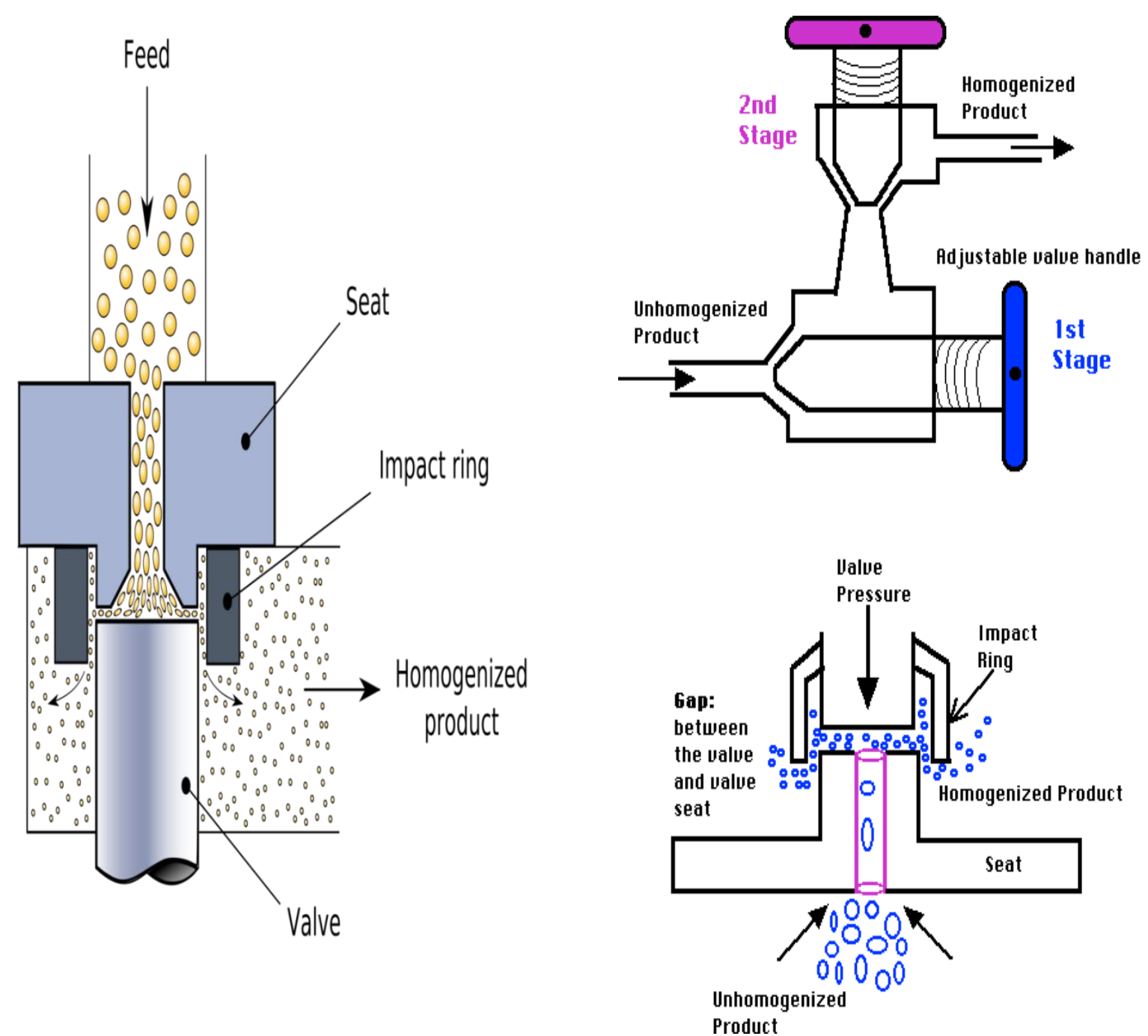

IV.

TWO-STAGE HOMOGENIZATION

The two-stage homogenizing valve (a Gaulin invention) has been available for many years. Experimental testing has suggested that the primary function of the second-stage valve is to influence intensity of the homogenization effect in the first stage.

Therefore, the second-stage valve in a sense "fine tunes" the homogenization process. For emulsions, the second-stage backpressure suppresses cavitation, eliminates two-phase flow and intensifies turbulence.

In oil-in-water emulsions, it has been found that the ideal second-stage pressure should be between 10 and $15 \%$ of the total homogenizing pressure. It has been demonstrated with milk that passage through the second-stage valve, itself, while maintaining a low backpressure as stated above, does not significantly alter the condition of the emulsion.

Sampling the milk both before and after the second-stage did not reveal any substantial change in emulsion quality. The second-stage valve is a convenient means for applying the correct backpressure to the first-stage homogenizing valve. A homogenizer processing milk at $2000 \mathrm{psi}$ (137.9 bar) with a two-stage valve may produce milk emulsion quality equivalent to a single-stage valve operating at 2200 to $2300 \mathrm{psi}$ (151.7 to 158.6 bar).

If the homogenizer has a maximum operating pressure of 2000 psi, then the two-stage valve configuration extends the achievable homogenization quality beyond that produced by a single-stage valve set at this maximum pressure.

There may be some products other than simple emulsions that have complex formulations that may be affected by the second-stage with respect to appearance or viscosity.

\subsection{1) Homogenizing Efficiency Measurements}


To determine the ideal operating pressure of the homogenizer on a particular product, the first step is to establish a method of evaluation. The method may be viscosity increase or decrease, average particle size (as determined microscopically or by instrument), rate of separation, grind gauge or change in physical appearance, just to mention a few. The next step is to collect samples from the homogenizer at different pressure settings; for example, every $1000 \mathrm{psi}$ (or less, if a narrow pressure range is being evaluated). Finally, using the appropriate method of analysis, the evaluated parameter is compared or plotted versus homogenizing pressure and the lowest pressure that generates the required product can then be selected. For some products it may be desirable to determine the effect of multipassing at an appropriate pressure.

[1] www.wikipedia.com

\section{REFERENCES}

[2] www.horiba.com

[3] http://www.nirosoavi.com/industries-applications/food-dairy-applications-homogenization.asp

[4] http://www.foodscience.uoguelph.ca/dairyedu/homogenization.html

[5] http://www.nirosoavi.com/industries-applications/homogenization-cosmetic-pharma-biotech.asp

[6] http://www.nirosoavi.com/industries-applications/homogenization-chemical-processing.asp

[7] http://www.raw-milk-facts.com/homogenization_T3.html

[8] http://www.proscientific.com/

[9] http://www.proscientific.com/Homogenizing.shtml

[10] http://www.wisegeek.com/what-is-homogenization.htm 